\title{
Development of a Drone-Supported Emergency Medical Service
}

\author{
Maria Elena Nenni ${ }^{1}$, Valentina Di Pasquale ${ }^{2}$, Salvatore Miranda², Stefano Riemma ${ }^{2}$ \\ ${ }_{1}^{1}$ Department of Industrial Engineering, University Federico II, Piazzale Tecchio, 80, 80125 Napoli, Italy \\ ${ }^{2}$ Department of Industrial Engineering, University of Salerno, Via Giovanni Paolo II, 132, 84084, SA, Italy
}

\begin{abstract}
There is a scientific consensus that the delivery of prompt emergency medical services (EMSs) guarantees a higher survival rate. An EMS is generally able to respond to $90 \%$ of higher priority calls in less than 9 minutes, with the best chance of survival being with a response time of 4-5 minutes. The major obstacle here is that a shorter response time would require the needed resources not to pass a certain threshold in a cost/benefit analysis. This paper aims to investigate the use of drones in as an EMS to improve response times. Although the literature already provides many examples of drones used for this purpose, they have all been developed as a prototype. This confirms the technical feasibility of a drone-based solution, but there is no evidence of the economic viability for such a service. The answer to this comes by analyzing the performance of an integratedwith-drones service as a whole. For this reason, we have redesigned the entire EMS model by including drones, and we have addressed the main issues, such as which types of service can be provided from drones, in which case, what the technical requirements for drones would be, and so on. Furthermore, we developed a specific procedure to keep the number of drones at a minimum level under the constraint of the minimum intervention time. The proposed model has been applied to a real EMS case for a city in the south of Italy. The outcome was that 96 drones were able to cover an area of 2,800 km², providing an intervention time of 4.5 minutes on average at an annual cost of less than $€ 300,000$. These results highlight that an integrated-with-drones service drastically improves the response time when compared with the traditional service, doing so at a viable cost.
\end{abstract}

Keywords: Cost/Benefit analysis; Design optimization; Drones; Emergency medical service

\section{Introduction}

There is a general need for an effective and timely response to emergencies (Dulebenets et al., 2019). Here, drones seem to fit well because they can be used for rescue missions (Yeong et al., 2015), environmental protection (Marris, 2013), and performing missions in oceans. One of the most promising sectors for developing drones is the healthcare field, where they can function in logistic operations and could be used for hospital deliveries (Roca-Riu and Menendez, 2019), even in remote areas (Tatsidou et al., 2019); indeed, one of the most important advantages of using drones is the potential to decrease the travel time for diagnosis and treatment (Laksham, 2019). That makes drones suitable for reducing the time and thus increasing the effectiveness of Emergency Medical Service (EMS). Hence, the current paper aims to investigate the use of drones in EMSs, which are, "a comprehensive system which provides the arrangements of personnel, facilities, 
and equipment for the effective, coordinated and timely delivery of health and safety services to victims of sudden illness or injury" (Moore, 1999)

The operations for an EMS include the process of a distress call on predefined protocols and translation into an alphanumeric priority code that includes the seriousness of the reported problem and location for the intervention. Based on these criteria, the most suitable and closest rescue vehicle is identified among those available to guarantee a timely and adequate response. The most common EMS performance measure is to respond to $90 \%$ of higher priority calls in less than 9 minutes (Fitch, 2005). Otherwise, the existing recommendations provided by medical and public safety experts typically advocate for 45 minutes for the response time (Pons et al., 2005). Although a shorter response time interval improves patient survival, covering most calls in less than 4 minutes tends to use resources in such a way that does not save the most patient lives overall (McLay, 2010). Clearly, a response time of 9 minutes is the result of a trade-off. In fact, a response time of 4-5 minutes would require such a certain amount of resources such as vehicles, staff and equipment, to not pass the costs and benefits. In the present paper, we intend to address two research statements.

\section{RS1: The response time drops to 4-5 minutes by using drones in the EMS}

Many prototypes that have already been tested have proven that drone use is an attainable goal, and no technological issues have emerged in their use. However, the performance of a prototype is one thing; integrating a fleet of drones in a real service is another matter, and it could affect the actual response time in many ways (availability of drones, effectiveness of the intervention, etc.). Accordingly, to give a complete answer to this question, we redesigned the entire EMS model by including drones and have addressed the issues coming from doing so, such as which kind of service can be provided from drones, in which case, the technical requirement for drones, and so forth. Addressed in such a way, RS1 also leads to the next research statement:

\section{RS2: An EMS service including drones is economically feasible}

An existing EMS could achieve a response time of 4-5 minutes just by using traditional emergency vehicles. The problem is that it would absorb so many resources to the point of making the service unfeasible and inefficient. So there must be a stronger reason to use drones in an EMS than simply because it is possible to do so. Indeed, here, drones can ensure better service in a viable way. Accordingly, our purpose is to evaluate the use of drones from an economical point of view, which is possible only after having integrated drones in an EMS to evaluate all the economic impacts of their use.

The present paper is organized as follows: Section 2 summarizes the most recent and relevant scientific contributions on using drones in EMSs. Section 3 is for developing specifications and then designing the new drones-supported EMS. Section 4 proposes a real application. Section 5 discusses the results from designing and applying the service. Finally, Section 6 presents our conclusions.

\section{Literature Review of Drones in EMS}

Rescue vehicles are divided into two large groups: terrestrial emergency vehicles and air rescue vehicles. The terrestrial emergency vehicles are typically ambulances. The air rescue vehicles are typically helicopters and drones. Despite the fact that the technology is relatively new, the literature displays many examples of applications for drones in EMSs. Specifically, for urban areas, a few papers have described the results from prototype applications. The majority of authors (Zègre-Hemsey et al., 2018) have decided to focus on ambulance drones for delivering defibrillators, and from their experiences, a drastically 
increased chance of survival for cardiac arrest patients emerges. Krishna et al. (2018) tried to develop a system that would be able to fly to the emergency spot earlier than an ambulance while taking into account multiple real-time health parameters of the patient, such as temperature, heart rate, and heartbeat. Dhivya and Premkumar (2017) developed an all-purpose ambulance drone consisting of a mini patient monitoring system that comprises variant sensors to measure important parameters and then send them to the ambulance, as well as to the nearby hospital, using a global positioning system.

However, drones are not meant to replace traditional emergency vehicles, and all the developed prototypes are limited to supporting ambulances. From previous studies' experiments conducted with prototypes, an answer to RQ1 can be found: performance is surely improved, and the chances of survival increased (Zègre-Hemsey et al., 2018). Nevertheless, in all the experiments, the focus has been on the performance of a single drone. In our opinion, the improvement should be evaluated by integrating a fleet of drones in an EMS. To do this, the entire service has to be redesigned, and drone specifications have to be clearly set. For these reasons, we investigated a second body of literature devoted to designing the service of drones. Scott and Scott (2017) reviewed the latest decision models that facilitate management decision making for operating a drone fleet. Then, in 2018, Troudi et al. (2018) tried to size the drone delivery fleet by considering energy autonomy. Kim et al. (2017) proposed two models for logistic arrangements of drones in aided healthcare service. Finally, Kartawijaya et al. (2019) tailored parameters, developed simulations for effective fleet size, and quantified an improved operator cost efficiency.

All these papers have addressed the economic feasibility of drones in an EMS and they have dealt with one or a few operational aspects. However, a systematic view is missing. This is why we have studied an entire service design for drones in an EMS.

\section{Design of a Drones-supported Emergency Medical Service}

The design of the service was developed based on the flowchart in Figure 1 . The starting point is selecting the disease to address because it is not possible to develop specifications for drones to be used in any kind of emergencies.

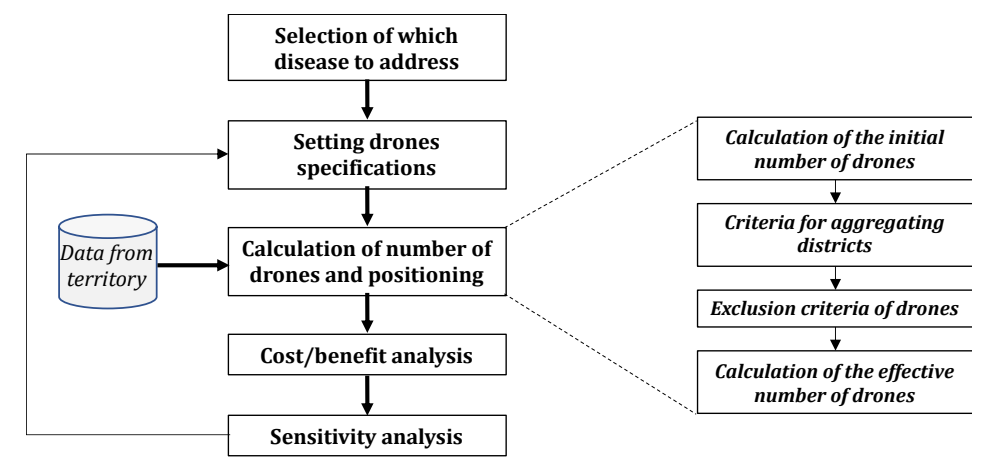

Figure 1 Flowchart for the design of a drone-supported emergency medical service

Almost all scholars have chosen to deal with the cardiac arrest emergency, specifically by making drones carry defibrillators. No specific evidence has been given to support this choice, but it is common sense: worldwide cardiovascular diseases are the leading cause of death. While acknowledging the soundness of this choice, we decided to further investigate it by analyzing the emergency calls recorded in the Italian city of Avellino in 2016, which is also the pilot case for the current paper. The emergency calls in 2016 totaled around 30,000; 54\% of which are most urgent (Priority 1 and Priority 2). From the bar chart in 
Figure 2 it is confirmed that the most recurring high priority intervention is for cardiological problems.

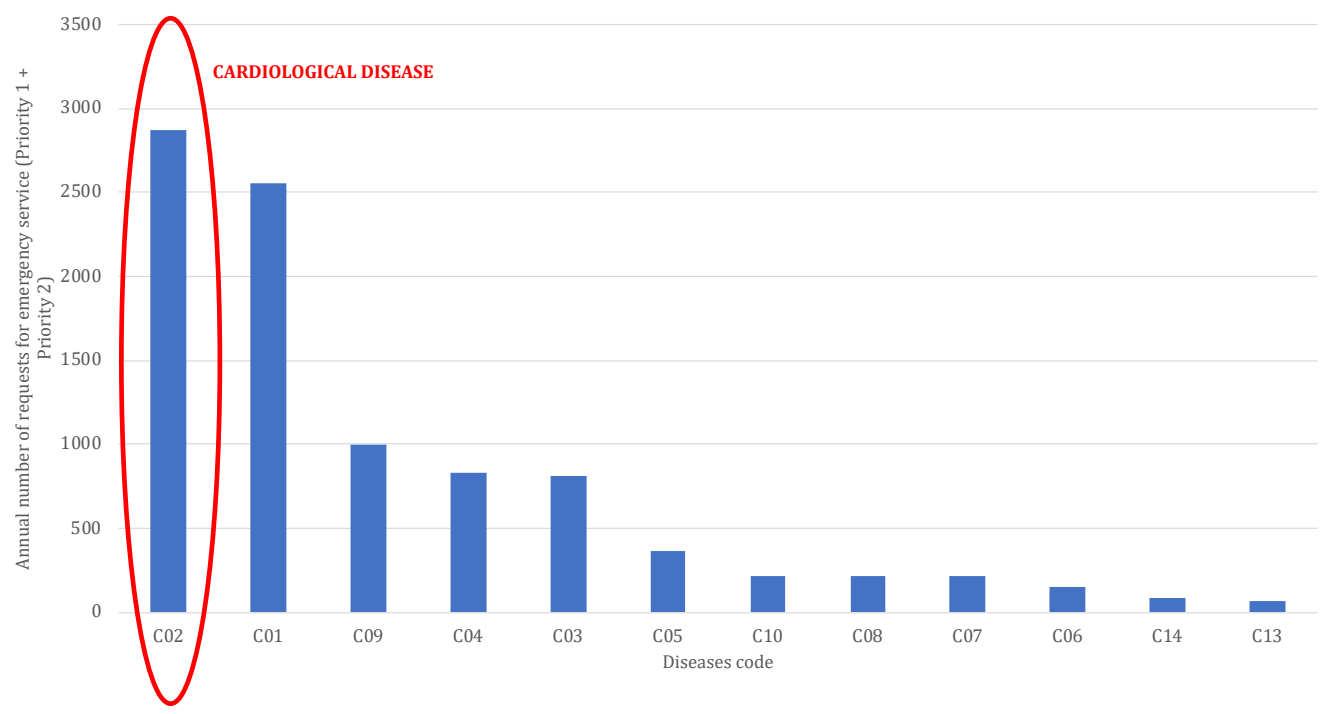

Figure 2 Annual number of requests for emergency service broken down by disease (Source: Local Health Department of Avellino, 2016)

\subsection{Setting Drones Specifications}

A drone should provide the appropriate diagnostic tools, such as an electrocardiograph and oximeter; tools necessary for the administration of the drugs; the defibrillator; portable early detection systems (Hugeng and Kurniawan, 2016); and all the medicine and equipment needed for first aid. A smartphone will allow the doctor to check on the patient's condition and support the first rescuer to use devices and medicines. Given the unit weight and quantity, it is possible to calculate the total weight of the trolley, which is lower than $4000 \mathrm{~g}$. These data are the first input to pick the most suitable type of drone.

Many drone classifications are available in the literature, each one using different drivers (size, ranges, etc.). We went for a taxonomy based on the maximum gross take-off weight (MGTW), which is the maximum all-up weight that the drone is permitted to take off at while being in compliance with its air-worthiness certification. According to Regulatory Article 1600, 2016, drones able to carry minimum gr 3268 fall into the NATO Class I and Military Aviation Authority (MAA) Class I(b) or Class I(c).

\subsection{Calculation of the Number of Drones}

When an emergency call arrives, the operations center contacts a control facility, which will then dispatch a suitable resource. In the case of a drone, the closest one to the emergency spot is identified, and the personnel prepares the drone, charging the cardiological trolley, and starts it toward the indicated GPS coordinates (Sutresman et al., 2017). Upon arrival, the drone will be taken over by the rescuer and later recovered by an ambulance, which will return it to the respective station. Therefore, the drone makes a oneway trip, fully loaded, toward the defined GPS coordinates.

A model to calculate the number of drones needed for the service is proposed below. To move from point $A$ to point $B$, the drone moves vertically to reach the defined altitude; then, it moves horizontally to reach point B; finally, it moves vertically to land at the defined point. Therefore, the variables to be considered are as follows:

- Horizontal speed with full load $\left(v_{h, f l}\right)$;

- Vertical speed with full load during take-off $\left(v_{t, f l}\right)$ and full load during landing $\left(v_{l, f l}\right)$; 
- Distance separating the drone's position from the location of the emergency $(d)$;

- Height reached from the drone to not face obstacles $\left(h_{t}\right)$ and during landing $\left(h_{l}\right)$;

- Corrective factor $(\alpha)$ : to take into account many different factors such as acceleration and deceleration, weather conditions, and so forth.

Once the variables are defined, the total time taken by the drone to make the journey $\left(t_{T O T}\right)$, also including the preparation time $\left(t_{\text {prep }}\right)$, is given by the following:

$$
t_{\text {TOT }}=t_{\text {prep }}+\left(\frac{d}{v_{h, f l}}+\frac{h_{t}}{v_{t, f l}}+\frac{h_{l}}{v_{l, f l}}\right) \cdot(1-\alpha)
$$

Given $t_{T O T}$, which must be lower than $4-5$ minutes as advocated by many experts, the maximum range of coverage that allows the drone to arrive on time is the following:

$$
r=\left[\frac{t_{\text {TOT }}-t_{\text {prep }}}{1+\alpha}-\frac{h_{t}}{v_{t, f l}}-\frac{h_{l}}{v_{l, f l}}\right] * v_{h, f l}
$$

Therefore, the radius of coverage $(r)$ is a function of the characteristics of the drone (speed), the characteristics of the territory to be covered (height), and the characteristics of the time taken for preparing the intervention.

Now, the number of drones $(\mathrm{N})$ needed to cover a specific territory is calculated accordingly to the following ratio:

$$
N=\frac{\text { Extent of a specific territory }}{\pi \cdot r^{2}}
$$

Typically, a territory is an area controlled or served from a control facility. In a mediumsized city, more control facilities can exist, each operating in a specific district.

Because one goal of the current research is to understand the economic feasibility of a drones-supported EMS, it makes sense to refer to a more extended area, that is, a province or even a region, depending on at which level the EMS is provided. This allows for aggregating districts, which would then be served by the same crew of drones. This is the point of the next step of the procedure, where the criteria to regroup different areas are proposed and the number of needed drones is recalculated.

Two or more districts can be grouped if all the following unification criteria are met:

- The number of theoretical drones for each of the considered districts is less than one;

- The distance between the districts is less than $r$ for the drone;

- The ratio between the sum of the extents of all the districts and the coverage area of the drone is always lower or, at most, equal to one.

An assessment of the height at which the drone must be taken is also required. By varying the height, the maximum distance that the drone can cover changes, as well as the number of drones. Once the aggregable districts have been identified, the number of drones needed to cover a given territory is recalculated.

Not all the districts require drones for the EMS, just those for which an intervention time of the ambulance is expected to be too much because of the distance from the facility to the place of the event or because of specific conditions, that is, traffic. Those presenting an expected rescue time compliance with these requirements do not need a drone-based EMS.

At this point in the procedure, all data and information to perform a standard cost/benefit analysis and a sensitivity analysis should be available. In the next section, we provide an example of an application for the entire procedure, as well as for the cost/benefit analysis and sensitivity analysis. 


\section{Application}

The proposed model has been applied to a real case by designing a drone service for the city of Avellino, which is a good test site because it has a good extension and areas at different heights, that are both major issues in using drones. In addition, we had full collaboration and commitment from the authority regarding the provision of data and information. Some data concerning the territory have been previously acquired. Specifically, because the city is divided into 118 districts, we first collected the air distance between each of the districts in a matrix. More districts sharing the same agency that are operating in a centralized way to provide dispatching services are then grouped in areas.

In Table 1, we report the distance matrix for a specific area we use as a quantitative example, even though we run the application for the whole city of Avellino. In addition, we acquired the average altitude of each district.

Table 1 Distance matrix for the Avellino area; average altitude; extension

\begin{tabular}{|c|c|c|c|c|c|c|c|c|c|c|c|}
\hline & 异 & 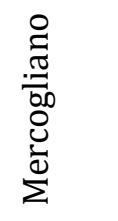 & 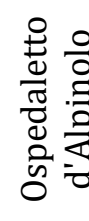 & 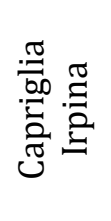 & 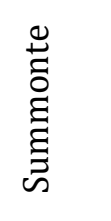 & $\begin{array}{l}\frac{\pi}{\pi} \\
\frac{\pi}{ \pm} \\
0 \\
0\end{array}$ & 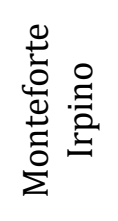 & $\begin{array}{l}\frac{\pi}{0} \\
\frac{0}{0} \\
\pm 0 \\
0 \\
0\end{array}$ & 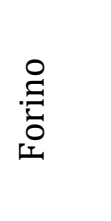 & 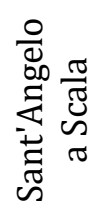 & 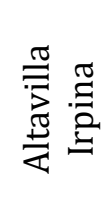 \\
\hline$\overline{\text { Avellino }}$ & - & 4.03 & 4.71 & 5.19 & 5.32 & $\overline{5.46}$ & $\overline{6.41}$ & 6.48 & 7.27 & 7.95 & 10.32 \\
\hline Mercogliano & 4.03 & & 1.93 & 5.18 & 3.03 & 6.98 & 3.90 & 6.79 & 6.63 & 5.93 & 10.01 \\
\hline $\begin{array}{l}\text { Ospedaletto } \\
\text { d'Alpinolo }\end{array}$ & 4.71 & 1.93 & - & 3.66 & 1.10 & 8.70 & 5.69 & 5.20 & 8.56 & 4.02 & 8.16 \\
\hline Capriglia Irpina & 5.19 & 5.18 & 3.66 & - & 2.95 & 10.50 & 9.07 & 1.61 & 11.40 & 3.59 & 5.20 \\
\hline Summonte & 5.32 & 3.03 & 1.10 & 2.95 & - & 9.68 & 6.77 & 4.37 & 9.66 & 2.96 & 7.10 \\
\hline Contrada & 5.46 & 6.98 & 8.70 & 10.50 & 9.68 & - & 5.84 & 11.90 & 3.63 & 12.62 & 15.69 \\
\hline Monteforte Irpino & 6.41 & 3.90 & 5.69 & 9.07 & 6.77 & 5.84 & & 10.68 & 3.56 & 9.46 & 13.85 \\
\hline Grottolella & 6.48 & 6.79 & 5.20 & 1.61 & 4.37 & 11.90 & 10.68 & - & 12.97 & 4.01 & 3.84 \\
\hline Forino & 7.27 & 6.63 & 8.56 & 11.40 & 9.66 & 3.63 & 3.56 & 12.97 & 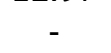 & 12.55 & 16.50 \\
\hline Sant'Angelo a Scala & 7.95 & 5.93 & 4.02 & 3.59 & 2.96 & 12.62 & 9.46 & 4.01 & 12.55 & - & 4.97 \\
\hline Altavilla Irpina & 10.32 & 10.01 & 8.16 & 5.20 & 7.10 & 15.69 & 13.85 & 3.84 & 16.50 & 4.97 & - \\
\hline$\overline{\text { Altitude (m a.s.l.) }}$ & 348 & 550 & 725 & 575 & 738 & 420 & 502 & 565 & 420 & 582 & 334 \\
\hline Extension $\left(\mathrm{Km}^{2}\right)$ & 30.41 & 19.76 & 5.62 & 7.38 & 12.44 & 10.31 & 26.70 & 7.12 & 20.49 & 10.48 & 14.10 \\
\hline
\end{tabular}

Table 2 Specifications for drones and other data for the model

\begin{tabular}{lc}
\hline \multicolumn{2}{c}{ Data } \\
\hline Total time for completing the service (sec) & 300 \\
Time for preparing the intervention (sec) & 60 \\
Horizontal speed with full load (m/s) & 16 \\
Vertical speed with full load during take-off $(\mathrm{m} / \mathrm{s})$ & 3 \\
Vertical speed with full load during landing $(\mathrm{m} / \mathrm{s})$ & 3 \\
Flight altitude $(\mathrm{m})$ & 50 \\
Corrective factor $(\alpha)$ & $20 \%$ \\
\hline
\end{tabular}

In Table 2, all the specifications for the drones and other data needed for the model are reported. The total time of the intervention was set at 5 minutes because a longer time would drastically reduce the probability of survival. Regarding the flight parameters of the drone, these refer to a commercial hexacopter (e.g. IF1200 model). The flight height was instead set at 50 meters above the ground. Finally, the correction coefficient $\alpha$ was set at 
$20 \%$ because being the province of Avellino is an Apennine area, the altitude is quite variable, even in the same area, so the drone is asked to often change its flight height. Based on these defined parameters, the maximum distance that each drone can cover is about 2.7 $\mathrm{km}$, while based on Formula 2, the coverage radius is $22.34 \mathrm{~km}$.

Finally, by Formula 3, it is possible to calculate the theoretical number of drones for each zone in the Avellino City (Table 3). In the example, 13 drones are needed. In total, for the entire city of Avellino, the number of drones required is 187.

It is now possible to apply the procedure to merge some districts and reduce the number of drones. In the selected area, the district of Mercogliano has a lower number of drones than the unit $\left(\mathrm{N}^{\circ}\right.$ of drones $=0.88$ ), so the possibility of merging can be assessed. The air distance from the other districts of the list was thus analyzed and sorted in ascending order. The first possibility of merging is with the district of Ospedaletto d'Alpinolo (1.93 $\mathrm{km})$.

Table 3 Number of drones in the selected area

\begin{tabular}{lcc}
\hline \multicolumn{1}{c}{ District } & $\mathrm{N}^{\circ}$ of drones & $\mathrm{N}^{\circ}$ of drones rounded up to the nearest integer \\
\hline Avellino & 1.36 & 2 \\
Mercogliano & 0.88 & 1 \\
Ospedaletto d'Alpinolo & 0.25 & 1 \\
Capriglia Irpina & 0.33 & 1 \\
Summonte & 0.56 & 1 \\
Contrada & 0.46 & 1 \\
Monteforte Irpino & 1.2 & 2 \\
Grottolella & 0.32 & 1 \\
Forino & 0.92 & 1 \\
Sant'Angelo a Scala & 0.47 & 1 \\
Altavilla Irpina & 0.63 & 1 \\
\hline
\end{tabular}

The total extension is 25.38 square kilometers, while the height difference between the two districts is 175 meters. Because this value is much higher than the flight height of the drone, it is necessary to recalculate the radius of the coverage of the drone, taking into account that the new height at which the drone must move is 225 meters (or 175 meters plus 50 meters, which represents the safest flight height accordingly to specifications in Table 2). The new coverage radius is about $2 \mathrm{~km}$ for merging two districts. Similarly, the zones of Capriglia and Grottolella can be merged and served with only one drone, as well as Summonte and Ospedaletto d'Alpinolo.

The total number of drones required to serve the area is now eight. Applying the same procedure to the entire city of Avellino, the total number of drones drops to 168. In accordance with what is reported in Section 3.2, some districts can be served by ambulance instead drone without any detriment in service. Specifically, we used as the parameters of choice the expected arrival time of ambulance and average road distance between from the ambulance station to the emergency site. Those parameters were set to 8 minutes and 5 $\mathrm{km}$, respectively. Applying this exclusion criterion, the number of effective drones necessary to cover the entire province of Avellino drops to 96.

\subsection{Sensitivity Analysis}

The number of drones calculated refers to the standard flight parameters (Table 2). By varying these values, the number of drones necessary to cover the entire province will change. A greater impact parameter is the horizontal speed with which the drone moves. 
The speed values considered were $50,60,70,80,90$, and $100 \mathrm{~km} / \mathrm{h}$ : for each of these values, the coverage radius and the number of corresponding drones are shown in Table 4 . The coverage radius varies linearly with speed, while the number of drones reacts in a nonlinear way. The theoretical number of drones rapidly decreases up to $70 \mathrm{~km} / \mathrm{h}$ and then tends to stabilize. This is because at least one drone is provided for each area. Therefore, an increase in drone speed drops only the number of drones in an area where more than one is present. The number of drones after merging continues to decrease with a slightly lower slope, even after $70 \mathrm{~km} / \mathrm{h}$. In fact, given the possibility of covering more areas with the same drone, an increase in speed involves even an increase in the possibility of merging more areas under the same drone.

Finally, the number of drones required decreases even more slowly with speed. This depends on the fact that the more the number of areas served by the drones decrease the less the merging procedure is applicable.

Table 4 Number of drones and coverage radius at variable speeds

\begin{tabular}{lcccccc}
\hline & $50 \mathrm{~km} / \mathrm{h}$ & $60 \mathrm{~km} / \mathrm{h}$ & $70 \mathrm{~km} / \mathrm{h}$ & $80 \mathrm{~km} / \mathrm{h}$ & $90 \mathrm{~km} / \mathrm{h}$ & $100 \mathrm{~km} / \mathrm{h}$ \\
\hline $\mathrm{N}^{\circ}$ of drones (theoretical) & 226 & 187 & 155 & 140 & 129 & 123 \\
$\mathrm{~N}^{\circ}$ of drones after grouping & 212 & 168 & 128 & 109 & 93 & 83 \\
$\mathrm{~N}^{\circ}$ of drones required & 126 & 96 & 75 & 66 & 58 & 52 \\
Coverage radius (m) & 2315 & 2667 & 3241 & 3704 & 4167 & 4630 \\
\hline
\end{tabular}

\subsection{Evaluation of the Use of Drones for Cases of Cardiological Pathology}

To make a comparison between the effectiveness of drones and ambulance service, we analyzed the calls for cardiological pathology that were recorded in 2016, here referring to requests for intervention from each of the 118 zones in the town of Avellino.

Approximately 4500 calls for cardiological pathology that were recorded in 2016 refer to requests for intervention, but only 1893 calls were considered because they were coming from areas that should be served by drones (source: Local Health Department of Avellino).

To perform a comparison, the estimated time of the intervention for the drone was calculated (Equation 1) and then compared with ambulance intervention time. Table 5 shows the results of this comparison for each district.

Table $5 \mathrm{~N}^{\circ}$ of calls, estimated intervention times-effective arrival time comparison

\begin{tabular}{|c|c|c|c|c|}
\hline District & & $\begin{array}{c}\mathrm{N}^{\circ} \text { of } \\
\text { emergency } \\
\text { calls }\end{array}$ & $\begin{array}{l}\text { Estimated intervention time } \\
\text { for drone (minutes) }\end{array}$ & $\begin{array}{l}\text { Average arrival time for } \\
\text { ambulance (minutes) }\end{array}$ \\
\hline Mercogliano & & 120 & 4.80 & 11 \\
\hline $\begin{array}{l}\text { Ospedaletto } \\
\text { d'Alpinolo }\end{array}$ & \} & 42 (in total) & 3.34 & 17 \\
\hline Summonte & & & 3.04 & 19 \\
\hline Capriglia Irpina & & & 3.58 & 17 \\
\hline Grottolella & \} & 40 (in total) & 3.67 & 22 \\
\hline $\begin{array}{l}\text { Sant'Angelo a } \\
\text { Scala }\end{array}$ & & 11 & 3.95 & 23 \\
\hline Altavilla Irpina & & 25 & 4.31 & 28 \\
\hline $\begin{array}{l}\text { Monteforte } \\
\text { Irpino }\end{array}$ & & 108 & 5.31 & 16 \\
\hline Forino & & 25 & 4.86 & 19 \\
\hline Contrada & & 20 & 3.93 & 13 \\
\hline
\end{tabular}


The same comparison was performed for all 1893 calls, leading to an average drone intervention time of 4-5 minutes against 15 minutes on average taken by ambulance.

\subsection{Cost Analysis}

In this section and based on data concerning emergency calls in 2016, we estimated the expected costs for covering the EMS with drones.

Concerning investments, we considered the cost of buying drones and accessories; cost to make the service operational; and the cost of the devices and medicines in the cardiological trolley. Concerning drones (Table 6), the cost of the unit has been taken as a reference to the cost of a hexacopter DIJ S900. Together with the number of drones to buy, we considered the cost for insurance (one for each drone) and battery (one plus a spare for each drone). Concerning the cost of the devices (one for each drone), they are reported in Table 7.

Because these are technological devices, amortization has been spread over three years. The cost of medicines has been estimated based on the quantity used from each specific disease, given the distribution of diseases among emergencies recorded in 2016 in Avellino City. The total estimated annual cost of the medicine is $€ 60,653.79$. In this numerical application, the total annual cost is €283,989.27. This number can be used as a baseline for evaluating the viability of the drone-supported EMS.

Table 6 Cost for drones and accessories

\begin{tabular}{lccc}
\hline \multicolumn{1}{c}{ Item } & Quantity & $\begin{array}{c}\text { Cost per } \\
\text { unit }(€)\end{array}$ & Cost (€) \\
\hline Drones & 96 & 2800 & 268,800 \\
Batteries & 192 & 56 & 10,752 \\
Insurance & 96 & 250 & 24,000 \\
\hline
\end{tabular}

Table 7 Cost for devices

\begin{tabular}{lccc}
\hline \multicolumn{1}{c}{ Item } & Quantity & $\begin{array}{c}\text { Cost per } \\
\text { unit }(€)\end{array}$ & Cost $(€)$ \\
\hline APPS & 1 & 20,000 & 20,000 \\
Tablet & 32 & 200 & 6,400 \\
Training & 96 & 1,000 & 96,000 \\
\hline
\end{tabular}

However, as a note, these are two completely different kinds of services because ambulances cover a longer list of emergencies and provide a more enhanced service. Otherwise, they cost a lot. According to Co.E.S Italia (Quanto costa un'ambulanza, 2020), the annual cost for an ambulance, including for insurance, personnel, and so forth is more than $€ 300,000$. With less money, a fleet of drones can cover the service for an entire town, limited only by disease coverage, but the most frequent one at that.

\section{Discussion of the Proposed Research Questions}

Concerning the first research statement, multiple sources (Zègre-Hemsey et al., 2018; Dhivya and Premkumar, 2017) proved that it is possible to drop the response time to 4-5 minutes. In addition, we developed a model to prove how it is possible to organize and provide an EMS service for an entire territory.

Concerning the second research statement, the issue was that even the traditional emergency vehicles such as ambulances are able to theoretically provide a response time of less than 5 minutes, but the cost makes this service largely unviable. The current paper tried to highlight how the cost seems to be pretty viable compared with the traditional service. In cases of cardiac arrest, the chances of survival decrease by $7-10 \%$ every minute. By analyzing the data of Avellino (area of 2,800 $\mathrm{km}^{2}$ ), we have seen that a fleet of 96 drones can provide the service in 4-5 minutes on average compared with the 15 minutes required from an ambulance to perform the same operation. In addition, in this case, the use of drones has entailed an expense of a few hundred thousand euros a year, which represents a sustainable expense compared with purchasing only a new ambulance. 


\section{Conclusions}

The main limitation of the present study is that we ran only one test for the model. However, given the extension of Avellino City and the characteristics of the territory, which are pretty rough, along with the many cases of heart disease per year, there is no reason to doubt comparable results, even in other cities.

Despite the obvious advantages, there are, however, factors limiting the use of drones as first aid tool, that is, the legal aspects and the readiness of the rescuer. The drone carries a first aid kit that must be used by the person who finds the patient in a critical state. He or she must have a tough attitude and must not be seized by emotions to properly follow the instructions indicated by the doctor. This, though, is an unpredictable factor.

Finally, we have addressed only the technical and economic feasibility in the current paper. According to the most recent literature on the cost/benefit analysis (Basten et al., 2019) and urban transportation (Nenni et al., 2019), it might be wise to assess the social and environmental feasibility of the service in future works.

\section{Acknowledgements}

The authors would like to thank Antonella D'Aquino for her help in implementing part of the model during her master's thesis in management engineering at the University of Salerno.

\section{References}

Basten, V., Crévits, I., Latief, Y., Berawi, M.A., 2019. Conceptual Development of Cost Benefit Analysis based on Regional, Knowledge, and Economic Aspects of Green Building. International Journal of Technology, Volume 10(1), pp. 81-93

Dhivya, A.J.A., Premkumar, J., 2017. Quadcopter-based Technology for an Emergency Healthcare. In: $3^{\text {rd }}$ International Conference on Biosignals, Images and Instrumentation (ICBSII)

Dulebenets, M.A., Pasha, J., Abioye, O. F., Kavoosi, M., Ozguven, E.E., Moses, R., Boot, W.R., Sando, T., 2019. Exact and Heuristic Solution Algorithms for Efficient Emergency Evacuation in Areas with Vulnerable Populations. International Journal of Disaster Risk Reduction, Volume 39, p. 101114

Fitch, J., 2005. Response Times: Myths, Measurement \& Management. JEMS: A Journal of Emergency Medical Services, Volume 30(9), pp. 47-56

Hugeng, H., Kurniawan, R., 2016. Development of the 'Healthcor' System as a Cardiac Disorders Symptoms Detector using an Expert System based on Arduino Uno. International Journal of Technology, Volume 7(1), pp. 78-87

Kartawijaya, T., Townsend, E., Tully, K., Isihara, P., Diedrichs, D. R., Flores, G., Ward, J., 2019. Is now the Time to Invest in Emergency Smart-navigated Multiple-response Quadcopter Fleets? Journal of Unmanned Vehicle Systems, Volume 7(2), pp. 145-155

Kim, S.J., Lim, G.J., Cho, J., Côté, M.J., 2017. Drone-aided Healthcare Services for Patients with Chronic Diseases in Rural Areas. Journal of Intelligent \& Robotic Systems, Volume 88(1), pp. 163-180

Krishna, V.V., Shastri, S., Kulshrestha, S., Mariajossy, M.A., 2018. Design of Drone Ambulance. International Journal of Pure and Applied Mathematics, Volume 119(15), pp. 18131818

Laksham, K.B., 2019. Unmanned Aerial Vehicle (Drones) in Public Health: A SWOT Analysis. Journal of Family Medicine and Primary Care, Volume 8(2), pp. 342-346 
McLay, L.A., 2010. Emergency Medical Service Systems that Improve Patient Survivability. Wiley Encyclopedia of Operations Research and Management Science. Available Online at https://onlinelibrary.wiley.com/doi/abs/10.1002/9780470400531.eorms0296

Marris, E., 2013. Drones in Science: Fly, and Bring me Data. Nature, Volume 498(7453), pp. 156-158

Moore, L., 1999. Measuring Quality and Effectiveness of Prehospital EMS. Prehospital Emergency Care, Volume 3(4), pp. 325-331

Nenni, M.E., Sforza, A., Sterle, C., 2019. Sustainability-based Review of Urban Freight Models. Soft Computing, Volume 23(9), pp. 2899-2909

Pons, P.T., Haukoos, J.S., Bludworth, W., Cribley, T., Pons, K.A., Markovchick, V.J., 2005. Paramedic Response Time: Does it Affect Patient Survival? Academic Emergency Medicine, Volume 12(7), pp. 594-600

Quanto costa un'Ambulanza del SSN e relativo Equipaggio di soccorso (How much an ambulance costs). Available Online at: http://www.coesitalia.eu/blog/delucidazionicosti/, Accessed on 12 February 2020

Regulatory Article (RA) 1600: remotely piloted air systems (RPAS). Available Online at: https://www.gov.uk/government/publications/regulatory-article-ra-1600-remotelypiloted-air-systems-rpas, Accessed on 12 February 2020

Roca-Riu, M., Menendez, M., 2019. Logistic Deliveries with Drones: State of the art of Practice and Research. In: 19th Swiss Transport Research Conference (STRC 2019). STRC, pp. 1-14

Scott, J., Scott, C., 2017. Drone Delivery Models for Healthcare. In: Proceedings of the 50 ${ }^{\text {th }}$ Hawaii International Conference on System Sciences

Sutresman, O., Syam, R., Asmal, S., 2017. Controlling Unmanned Surface Vehicle Rocket using GPS Tracking Method. International Journal of Technology, Volume 8(4), pp. 709718

Tatsidou, E., Tsiamis, C., Karamagioli, E., Boudouris, G., Pikoulis, A., Kakalou, E., Pikoulis, E., 2019. Reflecting upon the Humanitarian Use of Unmanned Aerial Vehicles (Drones). Swiss Medical Weekly, Volume 149(1314), pp. 1-6

Troudi, A., Addouche, S.A., Dellagi, S., Mhamedi, A.E., 2018. Sizing of the Drone Delivery Fleet Considering Energy Autonomy. Sustainability, Volume 10(9), pp. 1-17

Yeong, S.P., King, L.M., Dol, S.S., 2015. A Review on Marine Search and Rescue Operations using Unmanned Aerial Vehicles. International Journal of Mechanical, Aerospace, Industrial, Mechatronic and Manufacturing Engineering, Volume 9(2), pp. 396-399

Zègre-Hemsey, J.K., Bogle, B., Cunningham, C.J., Snyder, K., Rosamond, W., 2018. Delivery of Automated External Defibrillators (AED) by Drones: Implications for Emergency Cardiac Care. Current Cardiovascular Risk Reports, Volume 12(25), pp. 1-9 